\title{
Estimating the Effect of Intravenous Acetaminophen for Postoperative Pain Management on Length of Stay and Inpatient Hospital Costs
}

\author{
E. Eve Shaffer · An Pham • Robert L. Woldman • Andrew Spiegelman • \\ Scott A. Strassels · George J. Wan · Thomas Zimmerman
}

Received: September 7, 2016/Published online: November 9, 2016

(C) The Author(s) 2016. This article is published with open access at Springerlink.com

\section{ABSTRACT}

Introduction: The provision of safe, effective, cost-efficient perioperative inpatient acute pain management is an important concern among clinicians and administrators within healthcare institutions. Overreliance on opioid monotherapy in this setting continues to present health risks for patients and increase healthcare costs resulting from preventable adverse events. The goal of this study was to model length of stay (LOS), potential opioid-related complications, and costs for patients reducing opioid use and

Enhanced content To view enhanced content for this article go to http://www.medengine.com/Redeem/ BF07F0605CD22222.

E. E. Shaffer · R. L. Woldman · A. Spiegelman The Advisory Board Company, Washington, DC, USA

A. Pham $(\varangle) \cdot$ G. J. Wan

Mallinckrodt Pharmaceuticals, Hampton, NJ, USA

e-mail: an.pham@mallinckrodt.com

S. A. Strassels

Optum Hospice Pharmacy Services, St Louis, MO, USA

T. Zimmerman

Bernardsville, NJ, USA adding intravenous acetaminophen (IV APAP) for management of postoperative pain.

Methods: Data for this study were de-identified inpatient encounters from The Advisory Board Company across 297 hospitals from 2012-2014, containing 2,238,433 encounters (IV APAP used in $12.1 \%$ ). Encounters for adults $\geq 18$ years of age admitted for cardiovascular, colorectal, general, obstetrics and gynecology, orthopedics, or spine surgery were included. The effects of reducing opioids and adding IV APAP were estimated using hierarchical statistical models. Costs were estimated by multiplying modeled reductions in LOS or complication rates by observed average volumes for medium-sized facilities, and by average cost per day or per complication (LOS: US\$2383/day; complications: derived from observed charges).

Results: Across all surgery types, LOS showed an average reduction of $18.5 \%$ (10.7-32.0\%) for the modeled scenario of reducing opioids by one level (high to medium, medium to low, or low to none) and adding IV APAP, with an associated total LOS-related cost savings of $\$ 4.5 \mathrm{M}$. Modeled opioid-related complication rates showed similar improvements, averaging a 
reduction of $28.7 \%(5.4-44.0 \%)$ with associated cost savings of $\$ 0.2 \mathrm{M}$. In aggregate, costs decreased by an estimated $\$ 4.7 \mathrm{M}$ for a medium-sized hospital. The study design demonstrates associations only and cannot establish causal relationships. The cost impact of LOS is modeled based on observed data.

Conclusions: This investigation indicates that reducing opioid use and including IV APAP for postoperative pain management has the potential to decrease LOS, opioid-related complication rates, and costs from a hospital perspective.

Funding: Mallinckrodt Pharmaceuticals.

Keywords: Cost; Intravenous acetaminophen; IV APAP; Length of stay; Opioid; Opioid-related adverse drug events; ORADE; Pain; Pharmacoeconomic; Postoperative

\section{INTRODUCTION}

The provision of safe, effective, cost-efficient perioperative inpatient acute pain management is an important concern among clinicians and administrators within healthcare institutions. Across a variety of surgeries, $20-55 \%$ of patients report at least moderate pain and up to $75 \%$ report severe pain during the immediate postoperative period, for a mean duration ranging from 1 to 8 days [1-5]. While readmission rates after a surgical discharge may be low, pain is a common reason for readmission among those returning to the hospital [6]. Additionally, untreated or inadequately treated acute postoperative pain can lead to chronic postsurgical pain [7]. Furthermore, adverse drug events (ADEs) frequently are associated with postoperative pain management regimens. In general, ADEs have long been associated with increased mortality, longer hospital length of stay (LOS), and higher costs of hospitalization [8, 9].

In 2000, the United States (US) Joint Commission on Accreditation of Healthcare Organizations (JCAHO), now referred to as the Joint Commission (JC), introduced pain management standards aimed at upholding a patient's right to effective assessment and treatment of pain from admission to discharge [10]. However, the American Medical Association (AMA) has recommended that pain be removed as a "fifth vital sign" due to concerns about opioid over-prescribing, which critics contend could make it more difficult for those in pain to have their pain properly diagnosed and treated. Importantly, pain is a key input into the JC hospital accreditation score and the Hospital Consumer Assessment of Healthcare Providers and Systems (HCAHPS) CAHPS $^{\circledR}$ Hospital Survey, and can impact overall hospital reimbursement by the government.

\section{Acute Pain Management Using Opioids}

In the US, intravenous (IV) opioid agonists such as morphine, hydromorphone, and fentanyl have traditionally been important analgesics in the treatment of pain in the immediate perioperative and critical care settings [11-13]. In the first half of 2015 , over $70 \%$ of surgical inpatients and outpatients in the US received opioid monotherapy for acute pain management [14]. Overreliance on opioid monotherapy in the inpatient healthcare setting raises health risks for patients and increases healthcare costs resulting from preventable adverse events. Opioid monotherapy can significantly increase a variety of opioid-related adverse drug events (ORADEs), most commonly nausea, vomiting, urinary retention, fatigue, pruritus, sleep 
impairment, dizziness, sedation, somnolence, and headache [15]. Less commonly, opioid monotherapy has been associated with ileus [16], respiratory depression [17], falls, and delirium, and in some extreme cases even death [18]. Numerous studies have demonstrated that managing these ORADEs is costly and they are associated with increased LOS [19-22].

\section{Multimodal Analgesia}

As an alternative to opioid monotherapy, multimodal analgesia (MMA) captures the effectiveness of individual agents in optimal dosages that maximize efficacy and attempts to minimize side effects from one analgesic [7]. This important concept employs the theory that agents with different mechanisms of analgesia, such as nonopioids (acetaminophen and nonsteroidal anti-inflammatory drugs [NSAIDs]), opioids, local anesthetics, and anticonvulsants, may have synergistic effects in preventing or treating acute pain when used in combination [7]. The efficacy of multimodal analgesic regimens continues to improve, with opioid analgesics increasingly taking on the role of rescue analgesics for acute pain [23].

Multimodal analgesia has been shown to reduce ADEs, LOS, readmissions, and costs associated with the use of opioids in multiple surgical populations [24, 25]. Intravenous acetaminophen (IV APAP) has been used as a component of an MMA strategy to help contribute to reduced costs and improved outcomes. Although not all studies of perioperative use of IV APAP have demonstrated reductions in opioid use and LOS, recent economic impact studies have shown reduced costs associated with IV APAP $[26,27]$. These findings are supported by a pharmacoeconomic review of IV APAP, which concluded the body of evidence shows the drug has the potential to improve outcomes and hospital efficiency [28]. Up to an estimated 7\% of patients who receive prescription opioids following surgery end up using these medications long-term [29-33], which can result in tolerance, dependence, and addiction [34]. It remains to be demonstrated whether reductions in postoperative opioid consumption associated with IV APAP reduce the risk of inappropriate extended use of opioids.

The goal of the current study was to estimate, through the use of statistical modeling, LOS, potential opioid-related complications, and costs for patients reducing opioid use and adding IV APAP for management of postoperative pain.

\section{METHODS}

\section{Data Source}

Data for this retrospective cohort study were derived from de-identified hospital billing and administrative data from the Advisory Board Company. This comprised 2,238,433 inpatient encounters across 297 hospitals from 1 January 2012 through 31 December 2014, with IV APAP identified in 271,394 encounters (12.1\%). Hospitals were not segmented by size to derive parameter estimates; however, only medium-sized hospitals (100-399 beds) [35] were selected to determine the number of cases used to model cost impact.

Drug utilization (see Table 1 for a list of opioid and non-opioid generic drug names for which data were collected in this study) was identified using hospital charge data for the encounter and is subject to the typical limitations of this approach, such as the potential for coding errors, and charges may not equate to amount of drug administered. 
Table 1 Opioid and non-opioid drugs used in study

\begin{tabular}{|c|c|c|}
\hline Opioids & NSAIDs & Other non-opioids \\
\hline Alfentanil & Aspirin & Acetaminophen, injectable \\
\hline Buprenorphine & Celecoxib & Acetaminophen, non-injectable \\
\hline Buorphanol & Choline magnesium trisalicylate & Alpha-2 agonists (clonidine, dexmedetomidine) \\
\hline Codeine & Diclofenac & Gapabentinoids (gabapentin, pregabalin) \\
\hline Dihydrocodeine & Diflunisal & Local anesthetics, non-topical only (bupivacaine, \\
\hline Fentanyl & Etodolac & lidocaine, liposomal bupivacaine, ropivacaine) \\
\hline Hydrocodone & Fenoprofen & $N$-methyl-D-aspartate (NMDA) antagonists \\
\hline Hydromorphone & Flurbiprofen & (ketamine, memantine) \\
\hline Levorphanol & Ibuprofen & \\
\hline Meperidine & Indomethacin & \\
\hline Methadone & Ketoprofen & \\
\hline Morphine & Ketorolac & \\
\hline Nalbuphine & Magnesium salicylate/magnesium & \\
\hline Opium & salicylate tetrahydrate & \\
\hline Oxymorphone & Meclofenamate sodium & \\
\hline Paregoric & Mefenamic acid & \\
\hline Pentazocine & Meloxicam & \\
\hline Propoxyphene & Nabumetone & \\
\hline Remifentanil & Naproxen/naproxen sodium & \\
\hline Sufentanil & Oxaprozin & \\
\hline Tapentadol & Piroxicam & \\
\hline \multirow[t]{3}{*}{ Tramadol } & Salsalate & \\
\hline & Sulindac & \\
\hline & Tolmetin & \\
\hline
\end{tabular}

Opioids, non-steroidal anti-inflammatory drugs (NSAIDs) and other non-opioids used in this study are shown by generic name. Combination product names are not shown. Only data on intravenous acetaminophen are presented in this analysis

\section{Patient Inclusion and Exclusion Criteria}

Encounters for adults $\geq 18$ years of age admitted for cardiovascular, colorectal, general, obstetrics and gynecology (OBGYN), orthopedics, or spine surgery based on the encounters' All Patient Refined
Diagnosis-Related Group (APR-DRG) assignment (Table 2) were included. These six surgical categories represent high-volume procedures that were considered broadly applicable for the purpose of analysis.

Only data from acute care facilities with data available throughout the study period 
Table 2 APR-DRGs used in study

\begin{tabular}{ll}
\hline Category & Included APR-DRGs and APR-DRG codes \\
\hline Cardiovascular & Other vascular procedures (173) \\
& Percutaneous cardiovascular procedures with AMI (174) \\
& Percutaneous cardiovascular procedures without AMI (175) \\
Colorectal & Major small and large bowel procedures (221) \\
General & Appendectomy (225) \\
& Laparoscopic cholecystectomy (263) \\
OBGYN & Uterine and adnexa procedures for non-malignancy except leiomyoma (513) \\
& Cesarean delivery (540) \\
Vaginal delivery (560) & Hip joint replacement(301) \\
Orthopedic & Knee joint replacement (302) \\
& Hip and femur procedures for trauma except joint replacement (308) \\
& Knee and lower leg procedures except foot (313) \\
Shoulder, upper arm and forearm procedures (315) & Dorsal and lumbar fusion procedure except for curvature of back (304), intervertebral \\
disc excision and decompression (310), cervical spinal fusion and other back/neck \\
procedures excluding disc excision and decompression (321)
\end{tabular}

All Patients Refined Diagnosis Related Groups (APR-DRGs) by category and code used in this study are shown $A M I$ Acute myocardial infarction

and having sufficient data quality to identify drugs were included. Encounters with invalid age, gender, or severity, without diagnoses, without drug utilization, with mortalities or with discharge to hospice were excluded. LOS outliers were not removed from the analysis.

\section{Definition of Complications}

Potential ORADEs related to respiratory, gastrointestinal, central nervous system, urinary, and other events were defined using International Classification of Disease-9th Revision-Clinical Modification (ICD-9-CM) codes [20].

\section{Modeling and Analytical Approach}

There were two phases of modeling conducted in the study. The first phase was to determine parameter estimates, which describe the impact to LOS or complication rate based on drug utilization. The second was to employ those parameter estimates to model a specific treatment change and the downstream impact of that treatment change on LOS, complication rate, and costs. Because the data in this study were modeled, measures of central tendency and statistical tests of differences are not available.

The effects of reducing opioid use and adding IV APAP were estimated using 
hierarchical statistical models in Statistical Analysis Software $\left(\mathrm{SAS}^{\circledR}\right)$ 9.4. Independent variables analyzed were opioid use [none (no use)/low (1-3 estimated doses for 1 or 2 days)/ medium (4-8 estimated doses or 3 days' use)/ high (more than 8 estimated doses or greater than 3 days' use)], non-opioid use (none/low/ medium/high) and IV APAP use (none/used). Covariates were age, gender, Elixhauser comorbidity index, APR-DRG severity level, and admission type.

Stratified cluster sampling was conducted within each APR-DRG (sample size $~ 15,000$, with sample weights used to adjust for percent of each hospital encounter selected), and multiple replication studies were conducted to ensure robust results. Statistical modeling was used to determine parameter estimates for LOS and complications. Observed average LOS plus cost and charge data were used along with the parameter estimates to develop per-episode and per-facility cost impact estimates.

Cost impact estimates were generated by multiplying modeled reductions in LOS or complication rates by observed average volumes [facilities designated American Hospital Association (AHA) 100-399 beds], and by average cost per day of LOS or per complication [LOS: US\$2383/day (national benchmark from the Healthcare Cost and Utilization Project, HCUP, 2013), complications: derived from observed charges] (Tables 3, 4).

\section{Details of the Regression Modeling}

Hierarchical regressions were performed on random samples of $n=15,000$ from the full sample $(n=2,238,443)$. Specifically, separate regressions were performed for each APR-DRG (17 total) and each of those regressions had a random sample of $n=15,000$ from the given
APR-DRG. These regressions were replicated with additional $n=15,000$ samples to verify robustness of results and mitigate concerns about multiple comparisons. The levels used in hierarchical modeling were "facility" and "attending physician," with individual hospitalizations within those two levels.

The first outcome of interest was LOS in days, which was modeled using hierarchical linear regression on its natural logarithm. The second outcome of interest was complications, which were grouped into six binary variables based on the ICD-9-coded categories (respiratory, urinary, central nervous system, GI, general, and related) and modeled each using hierarchical logistic regression.

As predictors, the following were included: Opioids (ordinal, 0/1/2/3), non-opioids (ordinal, 0/1/2/3), IV APAP (binary, 0/1), patient gender, Elixhauser comorbidity score (continuous, 1-15, although it can theoretically be as high as 31), patient age, APR-DRG severity level (ordinal, 1-4), ICD-9-coded complications (binary, 0/1), admission type (categorical: "emergency," “urgent," “elective," “other"), and interactions between Elixhauser and each of (age, admission type, APR-DRG severity, and complications). Note that complications were predictors only in the LOS outcome models, and not in the complications outcome models. This article does not contain any new studies with human or animal subjects performed by any of the authors.

\section{RESULTS}

\section{Overall}

To put the impact of the LOS and complication rate reductions in context, the potential impact was modeled for a hypothetical 
Table 3 Sample calculations: LOS results (reducing one level of opioid use and adding IV APAP)

Variables

$\mathrm{CNT}=$ Observed average annual number of cases for medium-sized facilities (AHA, 100-399 beds)

$\mathrm{cCNT}=$ Observed average annual number of cases for medium-sized facilities (AHA, 100-399 beds) for all APR-DRG in the category

ALOS $=$ Observed average length of stay in days for the APR-DRG

cALOS $=$ Calculated average length of stay in days for the category

ALOSop $=$ Calculated average length of stay for reducing one level of Opioid use

ALOSiv $=$ Calculated average length of stay for reducing one level of Opioid use and adding IV APAP

cALOSiv $=$ Calculated average length of stay for reducing one level of opioid use and adding IV APAP for the category

Bop $=$ Opioid regression parameter

Biv $=$ IV APAP regression parameter

Cost per day of LOS (2013 HCUP): \$2383

APR-DRG level

ALOSop $=$ ALOS $-($ Bop $\times$ ALOS $)$ and ALOSiv $=$ ALOSop $+($ Biv $\times$ ALOSop $)$

Calculated LOS reduction $=$ ALOS - ALOSiv

$\%$ change of LOS reduction $=($ ALOS - ALOSiv $) /$ ALOS

Calculated per episode LOS cost savings $=($ ALOS - ALOSiv $) \times \$ 2383$

Calculated annual LOS cost savings (average medium-sized facility) $=($ ALOS - ALOSiv $) \times \$ 2383 \times$ CNT

Category level

$\mathrm{cCNT}=\mathrm{S}(\mathrm{CNT})$ and $\mathrm{cALOS}=\mathrm{S}(\mathrm{ALOS} \times \mathrm{CNT}) / \mathrm{cCNT}$

ALOSop $=$ ALOS $-($ Bop $\times$ ALOS $)$ and ALOSiv $=$ ALOSop $+($ Biv $\times$ ALOSop $)$

cALOSiv $=S($ ALOSiv $\times \mathrm{CNT}) / \mathrm{cCNT}$

Calculated LOS reduction for the category, rounded $=$ cALOS - cALOSiv

$\%$ change of LOS reduction for the category, rounded $=($ cALOS - cALOSvi $) / c A L O S$

Calculated annual LOS cost savings (average medium-sized facility),

rounded $=(\mathrm{cALOS}-\mathrm{cALOSiv}) \times \$ 2383 \times \mathrm{cCNT}$

Sample calculation methods used to model length of stay (LOS) results by reducing one level of opioid use and adding intravenous acetaminophen (IV APAP) are shown

medium-sized hospital. In aggregate, including both LOS- and complications-related reductions, annual costs for a medium-sized hospital were modeled to decrease by an estimated $\$ 4.7 \mathrm{M}$ by reducing opioid use and adding IV APAP for postoperative pain management in patients undergoing cardiovascular, colorectal, general, OBGYN, orthopedic, or spine surgery. Cost savings were driven by reductions in LOS and complication rates; the cost of IV APAP was not included in the analyses. A subgroup analysis was performed on outlier LOS, which demonstrated no overall impact on results. 
Table 4 Sample calculations: complications results (reducing one level of opioid use and adding IV APAP)

Variables

$\mathrm{CNT}=$ Observed average annual number of cases for medium-sized facilities (AHA, 100-399 beds)

$\mathrm{cCNT}=$ Observed average annual number of cases for medium-sized facilities (AHA, 100-399 beds) for all APR-DRG in the category

$\mathrm{ACR}=$ Observed average complication rate

$\mathrm{cACR}=$ Calculated average complication rate for the category

ACRop $=$ Calculated average complication rate for reducing one level of Opioid use

ACRiv $=$ Calculated average complication rate for reducing one level of Opioid use and adding IV APAP

cACRiv $=$ Calculated average complication rate for reducing one level of Opioid use and adding IV APAP for the category

$\mathrm{Bop}=$ Opioid regression parameter Biv $=$ IV APAP regression parameter

$\mathrm{CCR}=$ Typical cost-to-charge-ratio $=0.2$

$\mathrm{CPC}=$ Observed average charge increase per complication

$\mathrm{cCPC}=$ Observed average charge increase per complication for the category

$\mathrm{CST}=$ Charge-derived cost increase per complication c

$\mathrm{CST}=$ Charge-derived cost increase per complication for the category

APR-DRG level

$\mathrm{ACRop}=\mathrm{ACR}-(\mathrm{Bop} \times \mathrm{ACR})$ and $\mathrm{ACRiv}=\mathrm{ACRop}+(\mathrm{Biv} \times \mathrm{ACRop})$

Calculated complication rate reduction $=\mathrm{ACR}-\mathrm{ACRiv}$

$\%$ change of complication rate reduction $=(\mathrm{ACR}-\mathrm{ACRiv}) / \mathrm{ACR}$

$\mathrm{CST}=\mathrm{CPC} \times \mathrm{CCR}$

Reduction in complications $=(\mathrm{ACR}-\mathrm{ACRiv}) \times \mathrm{CNT}$

Calculated annual complication reduction cost savings (average medium-sized facility $)=($ ACR - ACRiv $) \times$ CNT $\times$ CST

Category level

$\mathrm{cCNT}=\mathrm{S}(\mathrm{CNT})$ and $\mathrm{cACR}=\mathrm{S}(\mathrm{ACR} \times \mathrm{CNT}) / \mathrm{cCNT}$

$\mathrm{ACRop}=\mathrm{ACR}-(\mathrm{Bop} \times \mathrm{ACR})$ and $\mathrm{ACRiv}=\mathrm{ACRop}+(\mathrm{Biv} \times \mathrm{ACRop})$

$\mathrm{cACRiv}=\mathrm{S}(\mathrm{ACRiv} \times \mathrm{CNT}) / \mathrm{cCNT}$

Calculated complication rate reduction $=\mathrm{cACR}-\mathrm{cACRiv}$

$\%$ change of complication rate reduction $=(\mathrm{cACR}-\mathrm{cACRiv}) / \mathrm{cACR}$

$\mathrm{CST}=\mathrm{CPC} \times \mathrm{CCR}$

$\mathrm{cCPC}=\mathrm{S}(\mathrm{CPC} \times \mathrm{CNT}) / \mathrm{cCNT}$

$\mathrm{cCST}=\mathrm{S}(\mathrm{CST} \times \mathrm{CNT}) / \mathrm{cCNT}$

Reduction in complications $=(\mathrm{cACR}-\mathrm{cACRiv}) \times \mathrm{cCNT}$ 
Table 4 continued

Calculated annual complication reduction cost savings (average medium-sized facility $)=($ cACR - cACRiv $) \times c C N T \times c C S T$

Sample calculation methods used to model complication results by reducing one level of opioid use and adding intravenous acetaminophen (IV APAP) are shown

\section{Length of Stay}

Across all surgery types, LOS showed an average of $18.5 \% / 1898.85$ cumulative total days reduction at the category level (categories ranging from $10.7 \% / 456.17$ total days to $32.0 \% / 161.12$ total days) for the modeled scenario of reducing opioid use by one level (high to medium, medium to low, or low to none) and replacing it with IV APAP, with an associated total LOS-related annual cost savings of $\$ 4.5 \mathrm{M}$ (Table 5). For example, the calculated annual impact for a medium-sized facility (admissions $\times$ LOS reductions $\times$ HCUP 2013 $\$ 2383$ cost per day of LOS) for the cardiovascular category overall (see first row in Table 5) is $276 \times 1.01 \times 2383=\$ 660,000$ (rounded). Of note, the absence of $P$ values and confidence intervals in Table 5 and Table 6 was due to derivation of cost savings estimates from a mix of nationally published reference costs and admission volumes, in addition to the parameter estimates from analysis of the observed data.

At the category level, general surgery showed the largest percentage reduction in LOS (32.0\%/total 161.12 days), while orthopedics, with much larger average case volume, showed the largest LOS-related cost reduction $(\$ 1,340,000)$. OBGYN showed the lowest percentage reduction in LOS $(10.7 \% / 456.17$ total days) (Table 5).

At the APR-DRG level, knee and lower leg procedures showed the largest percentage reduction in LOS (39.0\%/84.00 total days), closely followed by dorsal and lumbar fusion (34.5\%/137.16 total days) and laparoscopic cholecystectomy (34.3\%/123.48 total days), while cesarean delivery, with much larger average case volume, showed the largest LOS-related cost reduction $(\$ 600,000)$, despite having a low percentage reduction in LOS (13.5\%/253.44 total days). Vaginal delivery showed the lowest percentage reduction in LOS (7.5\%/167.62 total days), while uterine and adnexa procedures, with lower average case volume, showed the smallest LOS-related cost reduction $(\$ 80,000)$ (Table 5).

\section{Potential Opioid-Related Complications}

Reducing opioid use and adding IV APAP resulted in a reduction in the modeled opioid complication rate by an average of $28.7 \%$ (range 5.4-44.0\%), with associated complications-related annual cost savings of \$0.2 M (Table 6).

At the category level, spine showed the largest percentage reduction in complications (44.0\%) and the largest complications rate-related cost reduction $(\$ 70,000)$. The cardiovascular category showed the smallest percentage reduction in complications (5.4\%) and the smallest complications-related cost savings $(\$ 10,000)$, along with the OBGYN category (Table 6).

At the APR-DRG level, dorsal and lumbar spinal fusion showed the largest percentage reduction in complications (46.8\%), while bowel procedures showed the largest 


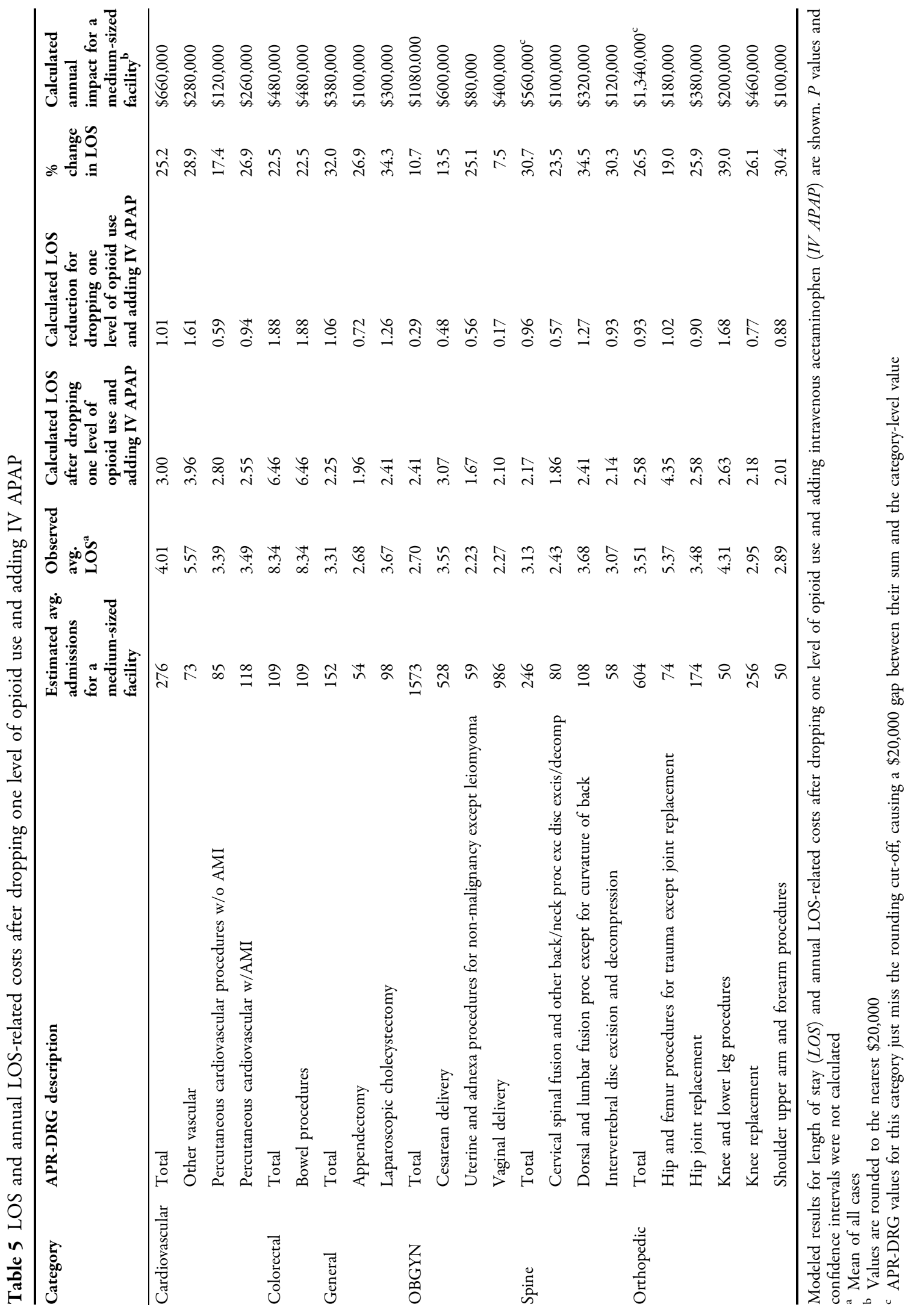




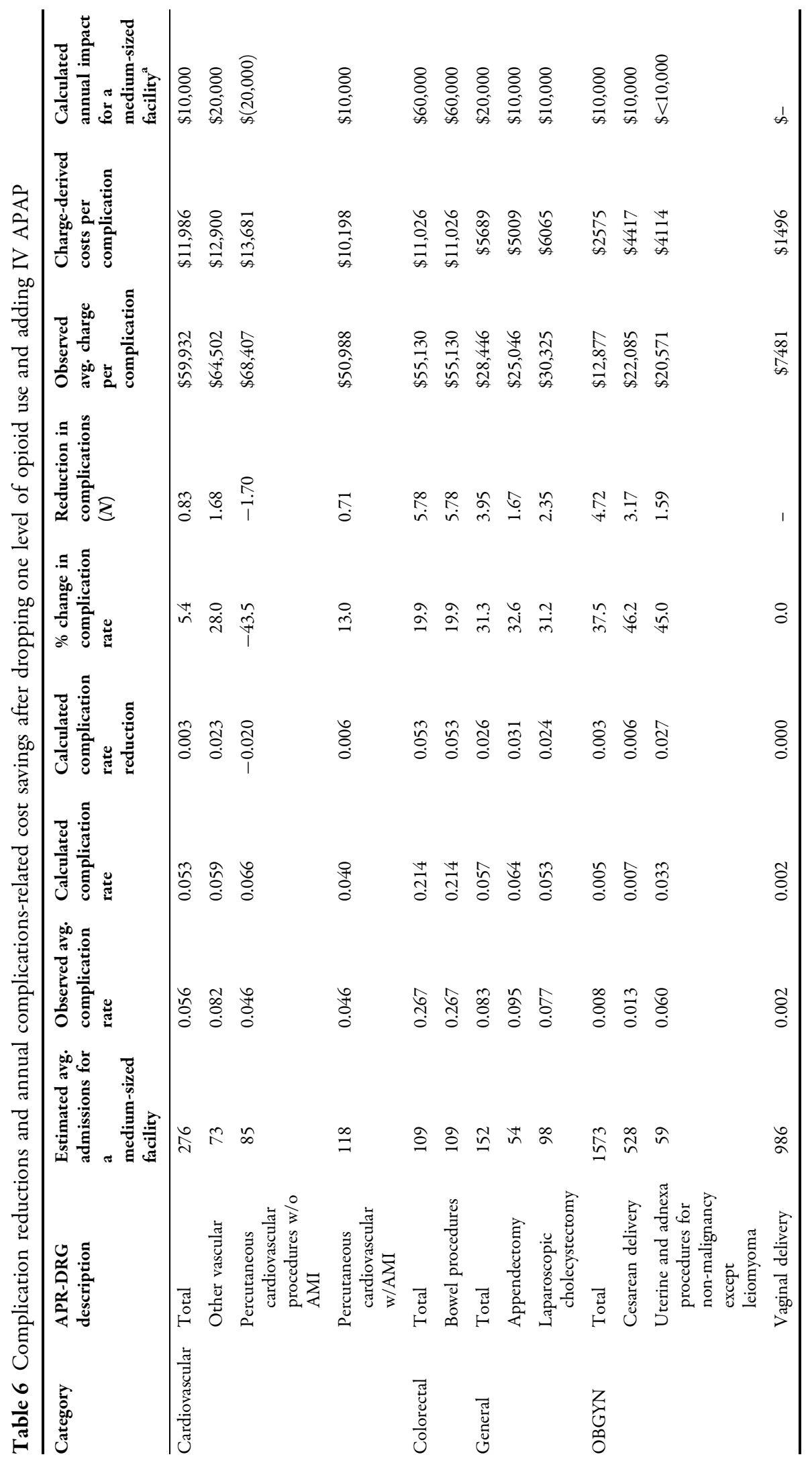




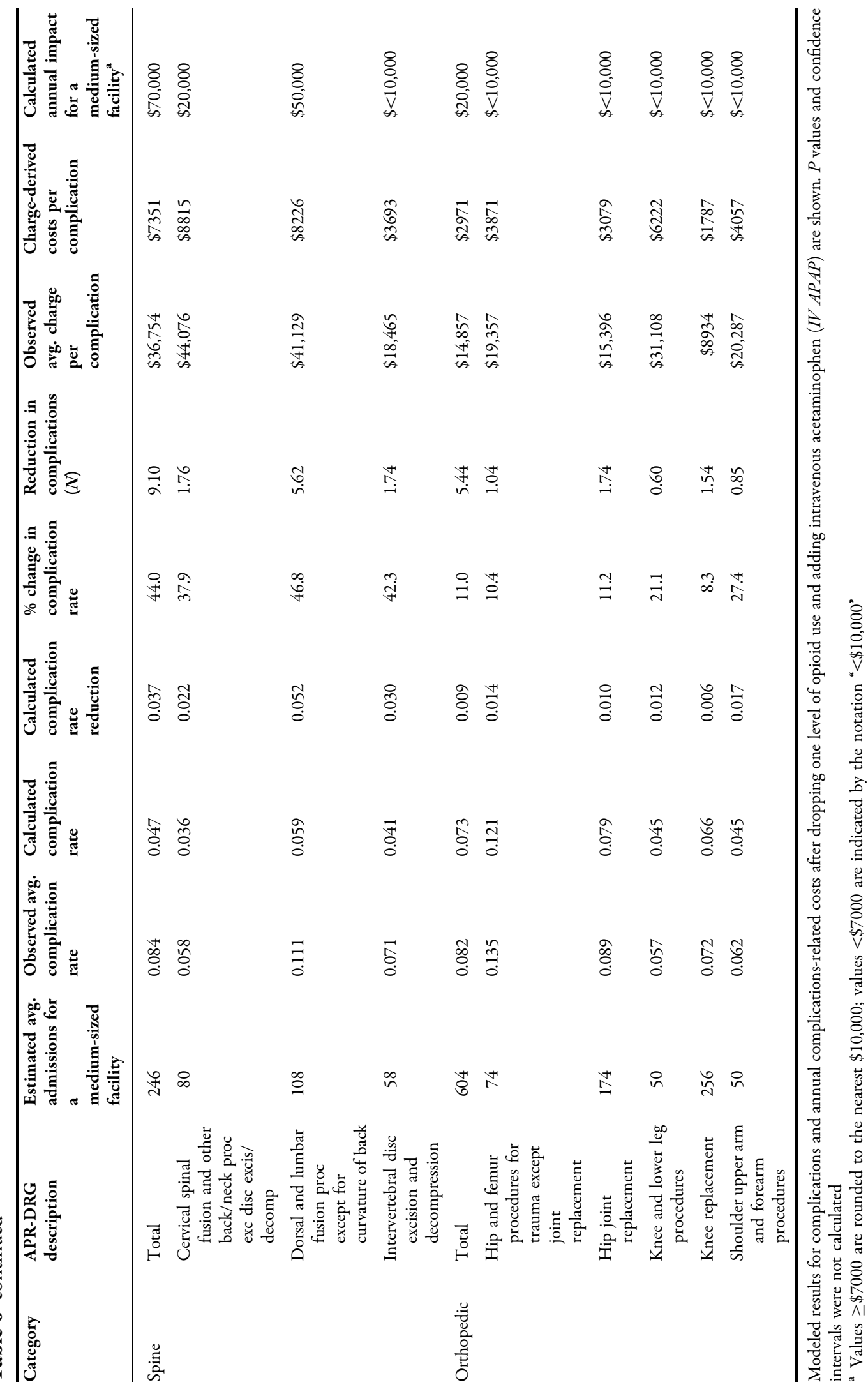


complications-related cost reduction $(\$ 60,000)$. Percutaneous cardiovascular procedures without acute myocardial infarction (AMI) showed the lowest percentage reduction in complication rate $(-43.5 \%)$, as well as the lowest complications-related cost reduction $(\$ 20,000)$-indicating that opioid reduction was modeled to have an adverse impact on complication rates for procedures in this APR-DRG. However, for the other 16 APR-DRGs, opioid reduction was modeled to have a positive impact on reducing complication rates for those procedures (Table 6).

\section{DISCUSSION}

While opioid therapy is a fundamental component of postoperative pain management, the risk of ADEs and negative patient outcomes pose significant safety concerns [36]. Increasingly, a multimodal approach to pain management is being used as a way to decrease postoperative opioid reliance but still provide optimal pain control [37]. Because of reduced opioid doses used, this new approach to pain management may decrease the risk of ORADEs [24, 38] and associated health care costs $[25,38]$. Recent pain guidelines for critically ill patients suggest this approach to avoid ADEs [12]. Since hospitals and outpatient clinics strive to provide care in an efficient and cost-effective manner to survive in the current budget-conscious healthcare climate, a major issue is providing balanced pain management while using the lowest effective dose of opioids. In particular, HCAHPS scores are tied to reimbursement, and two questions relate to pain management [39].

Prior studies have demonstrated postoperative use of IV APAP reduces opioid consumption. In the pivotal US study of IV APAP for acute pain management, IV APAP was associated with a $46 \%$ reduction over the first $6 \mathrm{~h}$ and 33\% reduction (vs. placebo) in total morphine consumption in the first $24 \mathrm{~h}$ following surgery [40]. Other randomized controlled trials (RCTs) have shown reductions in opioid consumption up to $78 \%$ [e.g., 41-45]. Taken together, results from prior studies [46-48] and the current analysis suggest a positive impact on overall hospital costs associated with reducing opioid consumption and adding IV APAP. Accordingly, IV APAP may be recommended as a standard of care in MMA regimens for postoperative pain management to reduce reliance on opioid monotherapy.

In RCTs, postoperative use of IV APAP has been associated with a shorter average LOS (0.4-1.5 days) relative to placebo or active controls [41, 45, 49]. In addition, a retrospective cohort study of case-matched patients who underwent total hip or knee replacement surgery [38], IV APAP used as part of an MMA strategy was associated with improved clinical outcomes in terms of fewer overall adverse events, shortened LOS, and reduced total hospital resources. A total of 22,146 cases and controls were similar in terms of age, race, sex, marital status, insurance status, and preoperative comorbidities. Overall adverse events were significantly lower with IV APAP (24.3\%) than with controls $(26.3 \%, P<0.001)$, numerically less frequent in all subgroups, and significantly less frequent for renal, infectious, and miscellaneous adverse events (all $P<0.05$ ). Also, IV APAP was associated with a shorter LOS, with 1 out of 11 patients discharged 1 day earlier $(P<0.001)$ and lower average hospital costs: $\$ 16,381$ for cases compared with $\$ 16,927$ for controls $(P<0.001)$. Cost savings estimated by structural equation modeling of $\$ 547$ per 
patient were due to $\$ 325$ from direct effects and $\$ 222$ from indirect effects, the latter mediated through adverse events and reduced LOS.

In the current study, the largest percentage reduction in LOS and opioid-related complications, seen in the spine category, may represent a greater benefit of adding IV APAP in more painful procedures. Specifically, spine surgery and other painful procedures generally require higher doses and/or more frequent administration of pain medications, with opioids used predominantly. Reducing the use of opioids in these patients by using IV APAP as part of an MMA strategy [50, 51] can reduce ORADE incidence and LOS $[47,52]$ while also reducing hospitalization costs [47].

In summary, the purpose of this study was to model the impact of reducing postoperative opioid use by implementing an MMA approach utilizing IV APAP. This investigation indicates that reducing opioid use and including IV APAP during treatment can contribute to decreased LOS, opioid-related complication rates, and costs from a hospital perspective. A subsequent sensitivity analysis restricting the data to the 24-h postoperative period was utilized to serve as an estimate of "perioperative" use on outcome measures. Results from this sensitivity analysis were similar to the current analysis.

\section{Limitations of the Study}

Although the authors consider the results of the current study to be valid, certain methodological procedures were not feasible to perform that would have enhanced rigor. Specifically, propensity scores or any other kind of pre-analysis matching data treatments were not used. In addition, study constraints precluded matching.

Opioid and non-opioid drug doses were categorized according to charge data, which has limited precision in regards to quantity/doses of drug a patient received. Therefore, ordinal bucketed categories were used instead of continuous measures. Estimated dosing was based on quantities charged, which is not a consistent representation of actual dosing. Out-of-range quantities were adjusted based on assumptions of use of $\mathrm{mg} / \mathrm{mL}$ as charge quantities rather than dose quantity.

Use of IV APAP was coded as a binary yes/no. Due to study constraints, as with opioid and non-opioid cases, a finer measure could not be used.

As listed in the regression details, some interactions were included whereas others were not. Optimally, the many dozens of different kinds of interactions between all our variables would have been examined, but, due to the large number of models involved in this study (17 APR-DRGs across 2 outcomes equals 34 models of interest), it was unfeasible to produce highly customized models for each case.

The limitations noted here raise the question whether all of the different modeling decisions made put the analysis at risk of implicit multiple comparisons problems $[53,54]$. While the data were not modeled in all of the variations desired, an attempt was made to mitigate the multiple concern by replicating regression results with new samples, as mentioned above. The replications performed also confirmed the findings of this analysis.

\section{Future Research Considerations}

Although the current study collected data on non-opioid pain medications in addition to IV APAP, analysis of these data was beyond the scope of the current study and may warrant future analysis and reporting. Additional work to better understand the impact of opioid 
reduction on percutaneous cardiovascular procedures without AMI may be of interest. Also, analysis of specific MMA approaches to identify the APR-DRGs for which they appear to provide the most benefit with respect to decreased LOS, opioid-related complication rates, and overall hospital costs may be warranted.

\section{CONCLUSIONS}

Prevention of ORADES rather than treatment of ADEs is a more effective strategy for hospitals, given the large economic burden and negative impact on patient outcomes associated with these events. A multimodal approach is a growing strategy to minimize the frequency of ORADEs as well as associated costs. This investigation indicates that reducing opioid use and including IV APAP for postoperative pain management has the potential to decrease LOS, opioid-related complication rates, and costs from a hospital perspective.

\section{ACKNOWLEDGEMENTS}

This research and the article processing charges were sponsored by Mallinckrodt Pharmaceuticals. Medical writing support was provided by Michael G. Baker, PhD, Samorn Biosciences, Inc. Support for this assistance was provided by Mallinckrodt Pharmaceuticals. All authors had full access to all of the data in this study and take full responsibility for the integrity of the data and accuracy of the data analysis. All named authors meet the International Committee of Medical Journal Editors (ICMJE) criteria for authorship for this manuscript, take responsibility for the integrity of the work as a whole, and have given final approval for the version to be published. These data were presented in part at the 2016 Perioperative Medicine Summit, February 25-27, 2016, Scottsdale, AZ, USA, and were selected for oral eposter presentation at the 41st Annual Regional Anesthesiology and Acute Pain Medicine Meeting, March 31-April 2, 2016, New Orleans, LA, USA.

Disclosures. E. Eve Shaffer is an employee of The Advisory Board Company. Robert L. Woldman is an employee of The Advisory Board Company. Andrew Spiegelman is an employee of The Advisory Board Company. George J. Wan is an employee of Mallinckrodt Pharmaceuticals, Inc. An Pham is an employee of Mallinckrodt Pharmaceuticals, Inc. Thomas Zimmerman is a former consultant to Mallinckrodt Pharmaceuticals. Scott A. Strassels is a former employee of Mallinckrodt Pharmaceuticals.

Compliance with Ethics Guidelines. This article does not contain any new studies with human or animal subjects performed by any of the authors.

Data Availability. The datasets generated during and/or analyzed during the current study available from the corresponding author on reasonable request.

Open Access. This article is distributed under the terms of the Creative Commons Attribution-NonCommercial 4.0 International License (http://creativecommons.org/licenses/ by-nc/4.0/), which permits any noncommercial use, distribution, and reproduction in any medium, provided you give appropriate credit to the original author(s) and the source, provide a link to the Creative Commons license, and indicate if changes were made. 


\section{REFERENCES}

1. Gan TJ, Habib AS, Miller TE, et al. Incidence, patient satisfaction, and perception of post-surgical pain: results from a US national survey. Curr Med Res Opin. 2014;30(1):149-60.

2. Gramke HF, de Rijke JM, van Kleef $M$, et al. The prevalence of postoperative pain in a cross-sectional group of patients after day-case surgery in a university hospital. Clin J Pain. 2007;23(6):543-8.

3. Joshi GP, Schug SA, Kehlet H. Procedure-specific pain management and outcome strategies. Best Pract Res Clin Anaesthesiol. 2014;28(2):191-201.

4. Mwaka G, Thikra S. Mung'ayi V. Prevalence of postoperative pain in the first 48 hours following day surgery at a tertiary hospital in Nairobi. Afr Health Sci. 2013;13(3):768-76.

5. Polomano RC, Dunwoody CJ, Krenzischek DA, et al. Perspective on pain management in the 21st century. J Perianesth Nurs. 2008;23:S4-14.

6. Coley KC, Williams BA, DaPos SV, et al. Retrospective evaluation of unanticipated admissions and readmissions after same day surgery and associated costs. J Clin Anesth. 2002;14:349-53.

7. Buvanendran A. Multimodal analgesia for perioperative pain management. IARS Review Course Lectures. 2011. http://www.iars.org/assets/ 1/7/11_RCL_Buvanendran.pdf. Accessed March 24, 2016.

8. Bates DW, Spell N, Cullen DJ, et al. The costs of adverse drug events in hospitalized patients. Adverse Drug Events Prevention Study Group. JAMA. 1997;277(4):307-11.

9. Classen DC, Pestotnik SL, Evans RS, et al. Adverse drug events in hospitalized patients: excess length of stay, extra costs, and attributable mortality. JAMA. 1997;277:301-6.

10. Phillips DM. JCAHO pain management standards are unveiled. JAMA. 2000;284(4):428.

11. ASA Task Force on Acute Pain Management. Practice guidelines for acute pain management in the perioperative setting: an updated report by the American Society of Anesthesiologists Task Force on Acute Pain Management. Anesthesiology. 2012;116:248-73.

12. Barr J, Fraser GL, Puntillo K, et al. Clinical practice guidelines for the management of pain, agitation, and delirium in adult patients in the intensive care unit. Crit Care Med. 2013;41:263-306.
13. Gandhi K, Baratta JL, Heitz JW, et al. Acute pain management in the postanesthesia care unit. Anesthesiol Clin. 2012;30(3):e1-15.

14. Premier Healthcare Alliance [paid-access hospital research database: data from January 2011-March 2015]. Charlotte: Premier, Inc.; 2015.

15. Zhao SZ, Chung F, Hanna DB, et al. Dose-response relationship between opioid use and adverse effects after ambulatory surgery. J Pain Symptom Manag. 2004;28:35-46.

16. Kehlet $\mathrm{H}$, Holte K. Review of postoperative ileus. Am J Surg. 2001;182(suppl 5A):3S-10S.

17. Cashman JN, Dolin SJ. Respiratory and haemodynamic effects of acute postoperative pain management: evidence from published data. $\mathrm{Br} \mathrm{J}$ Anaesth. 2004;93:212-23.

18. Benyamin R, Trescot AM, Datta S, et al. Opioid complications and side effects. Pain Physician. 2008;11(2 Suppl):S105-20.

19. Kessler ER, Shah M, Gruschkus SK, et al. Cost and quality implications of opioid-based postsurgical pain control using administrative claims data from a large health system: opioid-related adverse events and their impact on clinical and economic outcomes. Pharmacotherapy. 2013;33(4):383-91.

20. Oderda GM, Gan TJ, Johnson BH, et al. Effect of opioid-related adverse events on outcomes in selected surgical patients. J Pain Palliat Care Pharmacother. 2013;27(1):62-70.

21. Suh DC, Kim MS, Chow W, et al. Use of medications and resources for treatment of nausea, vomiting, or constipation in hospitalized patients treated with analgesics. Clin J Pain. 2011;27(6):508-17.

22. Pizzi LT, Toner R, Foley K, et al. Relationship between potential opioid-related adverse effects and hospital length of stay in patients receiving opioids after orthopedic surgery. Pharmacotherapy. 2012;32:502-14.

23. Elvir-Lazo OL, White PF. The role of multimodal analgesia in pain management after ambulatory surgery. Curr Opin Anesthesiol. 2010;23:697-703.

24. Maheshwari AV, Boutary M, Yun AG, et al. Multimodal analgesia without routine parenteral narcotics for total hip arthroplasty. Clin Orthop Relat Res. 2006;453:231-8.

25. Thiele RH, Rea KM, Turrentine FE, et al, Standardization of care: impact of an enhanced recovery protocol on length of stay, complications, 
and direct costs after colorectal surgery. J Am Coll Surg. 2015;220(4):430-43.

26. Subramanyam R, Varughese A, Kurth CD, Eckman $\mathrm{MH}$. Cost-effectiveness of intravenous acetaminophen for pediatric tonsillectomy. Paediatr Anaesth. 2014;24(5):467-75.

27. El Chaar M, Stoltzfus J, Claros L, Wasylik T. IV acetaminophen results in lower hospital costs and emergency room visits following bariatric surgery: a double-blind, prospective, randomized trial in a single accredited bariatric center. J Gastrointest Surg. 2016;20(4):715-24.

28. Jahr JS, Filocamo $\mathrm{P}$, Singh S. Intravenous acetaminophen: a review of pharmacoeconomic science for perioperative use. Am J Ther. 2013;20(2):189-99.

29. Alam A, Gomes T, Zheng H, Mamdani MM, Juurlink DN, Bell CM. Long-term analgesic use after low-risk surgery: a retrospective cohort study. Arch Intern Med. 2012;172:425-30.

30. Webster BS, Verma SK, Gatchel RJ. Relationship between early opioid prescribing for acute occupational low back pain and disability duration, medical costs, subsequent surgery and late opioid use. Spine. 2007;32:2127-32.

31. Sun EC, Darnall BD, Baker LC, Mackey S. Incidence of and risk factors for chronic opioid use among opioid-naive patients in the postoperative period. JAMA Intern Med. 2016;176(9):1286-93.

32. Huang A, Azam A, Segal S, et al. Chronic postsurgical pain and persistent opioid use following surgery: the need for a transitional pain service. Pain Manag. 2016. [Epub ahead of print].

33. Inacio MC, Hansen C, Pratt NL, Graves SE, Roughead EE. Risk factors for persistent and new chronic opioid use in patients undergoing total hip arthroplasty: a retrospective cohort study. BMJ Open. 2016;6(4):e010664.

34. Dowell D, Haegerich TM, Chou R. CDC guideline for prescribing opioids for chronic pain-United States, 2016. MMWR Recomm Rep. 2016;65(1):1-49.

35. American Hospital Association (AHA). AHA data viewer-glossary. https://www.ahadataviewer.com/ glossary/. Accessed August 1, 2016.

36. Swegle JM, Logemann C. Management of common opioid-induced adverse effects. Am Fam Physician. 2006;74(8):1347-54.

37. Voepel-Lewis T, Wagner D, Burke C, et al. Early adjuvant use of nonopioids associated with reduced odds of serious postoperative opioid adverse events and need for rescue in children. Paediatr Anaesth. 2013;23(2):162-9.

38. Apfel C, Jahr JR, Ernst FR, et al. Effect of intravenous acetaminophen on total hip or knee surgery: a case-matched evaluation of a national hospital patient record database. Am J Health Syst Pharm. 2015;72:1961-8.

39. Centers for Medicare and Medicaid. HCAHPS: patients' perspectives of care survey. https://www. cms.gov/medicare/quality-initiatives-patient-assess ment-instruments/hospitalqualityinits/downloads/ hospitalhcahpsfactsheet201007.pdf. Accessed March 24, 2016.

40. Sinatra RS, Jahr JS, Reynolds LW, et al. Efficacy and safety of single and repeated administration of 1 gram intravenous acetaminophen injection (paracetamol) for pain management after major orthopedic surgery. Anesthesiology. 2005;102:822-31.

41. Abdelmageed WM, Al Taher WM. Preoperative paracetamol infusion reduces sevoflurane consumption during thyroidectomy under general anesthesia with spectral entropy monitoring. Egyptian J Anaesth. 2014;30:115-22.

42. Arici S, Gurbet A, Türker G, et al. Preemptive analgesic effects of intravenous paracetamol in total abdominal hysterectomy. Agri. 2009;21(2):54-61.

43. Atef A, Fawaz AA. Intravenous paracetamol is highly effective in pain treatment after tonsillectomy in adults. Eur Arch Otorhinolaryngol. 2008;265(3):351-5.

44. Jokela R, Ahonen J, Seitsonen E, et al. The influence of ondansetron on the analgesic effect of acetaminophen after laparoscopic hysterectomy. Clin Pharm Ther. 2010;87:672-8.

45. Smith AN, Hoefling VC. A retrospective analysis of intravenous acetaminophen use in spinal surgery patients. Pharmacy Pract. 2014;12(3):417-22.

46. Hansen RN, Pham A, Balaban S, et al. Comparative analysis of inpatient costs for obstetrics and gynecology surgery patients treated with intravenous (IV) acetaminophen plus opioids or IV opioids alone for postoperative pain [abstract]. J Women's Health. 2016;25(4):A15.

47. Hansen RN, Pham A, Strassels SA, Balaban S, Wan GJ. Comparative analysis of length of stay and inpatient costs for orthopedic surgery patients treated with IV acetaminophen and IV opioids vs. IV opioids alone for post-operative pain. Adv Ther. 2016;33(9):1635-45. 
48. Shah MV, Maiese BA, Eaddy MT, et al. Hospitalization costs for patients undergoing orthopedic surgery treated with intravenous acetaminophen (IV-APAP) + IV opioids or IV opioids alone for postoperative pain. Annual Congress of Enhanced Recovery and Perioperative Medicine, April 20-22, 2016, Washington, DC, Poster presentation.

49. Looke TD, Kluth CT. Effect of preoperative intravenous methocarbamol and intravenous acetaminophen on opioid use after primary total hip and knee replacement. Orthopedics. 2013;36(2 Suppl):25-32.

50. Smith AN, Hoefling VC. A retrospective analysis of intravenous acetaminophen use in spinal surgery patients. Pharm Pract (Granada). 2014;12(3):417.

51. Mathiesen O, Dahl B, Thomsen BA, et al. A comprehensive multimodal pain treatment reduces opioid consumption after multilevel spine surgery. Eur Spine J. 2013;22(9):2089-96.
52. Walid MS, Hyer L, Ajjan M, Barth AC, Robinson JS Jr. Prevalence of opioid dependence in spine surgery patients and correlation with length of stay. J Opioid Manag. 2007;3(3):127-8, 130-2.

53. Gelman A, Loken E. The garden of forking paths: Why multiple comparisons can be a problem, even when there is no "fishing expedition" or "p-hacking" and the research hypothesis was posited ahead of time. Department of Statistics, Columbia University, New York. http://www.stat. columbia.edu/ gelman/research/unpublished/p_ hacking.pdf.

54. Simmons JP, Nelson LD, Simonsohn U. False-positive psychology: undisclosed flexibility in data collection and analysis allows presenting anything as significant. Psychol Sci. 2011;22(11):1359-66. 